\author{
Kacper Topolnicki · Jacek Golak • Roman Skibiński • \\ Henryk Witała
}

\title{
Three Nucleon Scattering in a 3D Approach at the First Order
}

Received: 19 October 2016 / Accepted: 18 November 2016 / Published online: 16 December 2016

(C) The Author(s) 2016. This article is published with open access at Springerlink.com

\begin{abstract}
The "three dimensional" (3D) approach to few-nucleon calculations, instead of working with partialwave states, uses the momentum degrees of freedom of the nucleons directly. As a result, 3D calculations are equivalent to incorporating all partial waves into the calculation. This equivalence is limited by the available computational resources, nonetheless, the new approach provides an opportunity to construct numerical descriptions of physical systems that were previously unavailable with more traditional methods. We present selected observables for the neutron-deuteron inelastic scattering process calculated using the 3D approach and the traditional partial wave approach. This comparison was carried out using the first order terms of the series expansion of the Faddeev equations and is a strong motivator for a development of a complete 3D calculation that utilizes all orders.
\end{abstract}

\section{Introduction}

Our description of three nucleon (3N) scattering is based on the Faddeev equation [1,2]:

$$
\check{T}=\check{t} \check{P}+\check{t} \check{G}_{0} \check{P} \check{T}
$$

where the inverted hat ${ }^{2}$ is used to mark operators and distinguish them from unit vectors typically denoted by a hat ${ }^{\wedge}, \breve{G}_{0}$ is the free $3 \mathrm{~N}$ propagator, $\check{t}$ is the two-nucleon $(2 \mathrm{~N})$ transition operator satisfying the LippmannSchwinger equation and $\check{P}=\check{P}_{12} \check{P}_{23}+\check{P}_{13} \check{P}_{23}$ is a permutation operator composed from particle exchange operators $\check{P}_{i j}$. Finally $\check{T}$ is the $3 \mathrm{~N}$ transition operator that can be used to calculate observables in both the elastic and breakup channels of the nucleon-deuteron scattering process.

In [3] we present a comparison of results based on partial waves and 3D results for observables related to the neutron induced deuteron breakup reaction and to elastic neutron deuteron scattering. This comparison suggests that the traditional calculations encounter convergence problems for certain kinematical regimes and that the new formalism can be used to obtain accurate observables in these cases. Although the results presented in [3] are obtained using only first order terms of the series expansion of the Faddeev equation (1) with $\check{T}=\check{t} \check{P}$, this comparison serves as a big motivator for the further development of the 3D approach.

This article belongs to the Topical Collection "The 23rd European Conference on Few-Body Problems in Physics".

K. Topolnicki $(\varangle) \cdot$ J. Golak $\cdot$ R. Skibiński $\cdot$ H. Witała

M. Smoluchowski Institute of Physics, Jagiellonian University, 30348 Kraków, Poland

E-mail: kacper.topolnicki@uj.edu.pl 


\section{Formalism}

Restricting the calculation to the first order with $\check{T}=\check{t} \check{P}$ makes it possible to calculate all observables in the breakup channel of $n d$ scattering from simple matrix elements [1,3]:

$$
\left\langle\phi_{0}|(\check{1}+\check{P}) \check{t} \check{P}| \phi\right\rangle
$$

and in the elastic channel from:

$$
\left\langle\phi^{\prime}\left|\check{P} \check{G}_{0}^{-1}+\check{P} \check{t} \check{P}\right| \phi\right\rangle .
$$

In (2) and (3) $|\phi\rangle,\left|\phi^{\prime}\right\rangle$ are states containing the deuteron bound state in the subspace of particles 2, 3 with the remaining particle being a free neutron and $|\phi\rangle_{0}$ is a state with three free nucleons.

The $2 \mathrm{~N}$ transition operator $\breve{t}$ appearing in (1-3) is a crucial element of the calculation. In our "three dimensional" treatment of $3 \mathrm{~N}$ scattering this operator is calculated using methods from [4,5]. First, the $2 \mathrm{~N}$ potential and transition operator are written in their general forms that explicitly obey the usual symmetries of parity, time reversal, particle exchange and spatial rotations [6]:

$$
\begin{aligned}
&\left\langle t^{\prime} m_{t}^{\prime} \boldsymbol{p}^{\prime}|\check{V}| t m_{t} \boldsymbol{p}\right\rangle=\sum_{i=1}^{6} v_{i}^{t m_{t}}\left(\left|\boldsymbol{p}^{\prime}\right|,|\boldsymbol{p}|, \hat{\boldsymbol{p}}^{\prime} \cdot \hat{\boldsymbol{p}}\right) \check{w}_{i}\left(\boldsymbol{p}^{\prime}, \boldsymbol{p}\right), \\
&\left\langle t^{\prime} m_{t}^{\prime} \boldsymbol{p}^{\prime}|\check{t}| t m_{t} \boldsymbol{p}\right\rangle=\sum_{i=1}^{6} \tau_{i}^{t m_{t}}\left(E ;\left|\boldsymbol{p}^{\prime}\right|,|\boldsymbol{p}|, \hat{\boldsymbol{p}}^{\prime} \cdot \hat{\boldsymbol{p}}\right) \check{w}_{i}\left(\boldsymbol{p}^{\prime}, \boldsymbol{p}\right),
\end{aligned}
$$

where $\boldsymbol{p}\left(\boldsymbol{p}^{\prime}\right)$ is the initial (final) relative momentum of the $2 \mathrm{~N}$ system. The isospin in the initial (final) state is $t\left(t^{\prime}\right), m_{t}\left(m_{t}^{\prime}\right)$ is the isospin projection in the initial (final) state and $E$ is the energy. Since $\check{w}_{i}\left(\boldsymbol{p}^{\prime}, \boldsymbol{p}\right)$ are given operators in the spin space (listed for example in [5]), the potential and transition operators in (4), (5) are effectively defined by the scalar functions $v_{i}^{t m_{t}}\left(\left|\boldsymbol{p}^{\prime}\right|,|\boldsymbol{p}|, \hat{\boldsymbol{p}}^{\prime} \cdot \hat{\boldsymbol{p}}\right)$ and $\tau_{i}^{t m_{t}}\left(E ;\left|\boldsymbol{p}^{\prime}\right|,|\boldsymbol{p}|, \hat{\boldsymbol{p}}^{\prime} \cdot \hat{\boldsymbol{p}}\right)$, respectively. This can be used in the next step to transform the Lippmann-Schwinger equation for $\check{t}$ :

$$
\check{t}=\check{V}+\check{V} \check{G}_{0} \check{t}
$$

into a set of coupled linear equations for the scalar functions $\tau_{i}^{t m_{t}}\left(E ;\left|\boldsymbol{p}^{\prime}\right|,|\boldsymbol{p}|, \hat{\boldsymbol{p}}^{\prime} \cdot \hat{\boldsymbol{p}}\right)$. Details on this procedure can be found in $[4,5]$.

Different numerical methods can be used to solve the resulting linear equations. In practical calculations we use Krylov subspace algorithms and in particular the Arnoldi algorithm [7] to turn the problem into a matrix equation of manageable size. The resulting scalar functions are plugged into (5) which is in turn used to calculate the matrix elements (2), (3) needed to construct the observables. Using (5) instead of utilizing the partial wave decomposition of $t$ results in a greater precision at larger energies since higher partial waves constructed from heavily oscillating functions are not needed. In the next section we show selected results from our paper [3] that illustrate this property.

\section{Numerical Results and Summary}

Out of the observables presented in [3] the numerical results for neutron induced deuteron breakup at laboratory kinetic energy $190 \mathrm{MeV}$ best illustrate the grater precision of 3D calculations. These results are presented in Fig. 1 for a chosen kinematical configuration in the final state [3] and show the convergence of the partial wave results to the $3 \mathrm{D}$ results with an increasing number of partial waves included in the calculation. Full agreement with the $3 \mathrm{D}$ results is not obtained since including more partial waves exceeded the capabilities of the computational resources used in the calculation. Additionally, since the construction of higher partial waves requires heavily oscillating functions, it is not obvious that such an increase would lead to full agreement using current numerical methods.

We conclude that constructing 3D calculations that utilize all orders of the Faddeev equation (1) might make it possible to describe physical situations that are currently unavailable for more traditional methods. Our recent work suggests that this goal can be achieved by constructing a general operator form of the scattering state $\check{T}|\phi\rangle$, where this state is effectively defined by a set of scalar functions. Progress in this direction has been made using the procedure from [8] for the construction of the general form of operators that obey given 

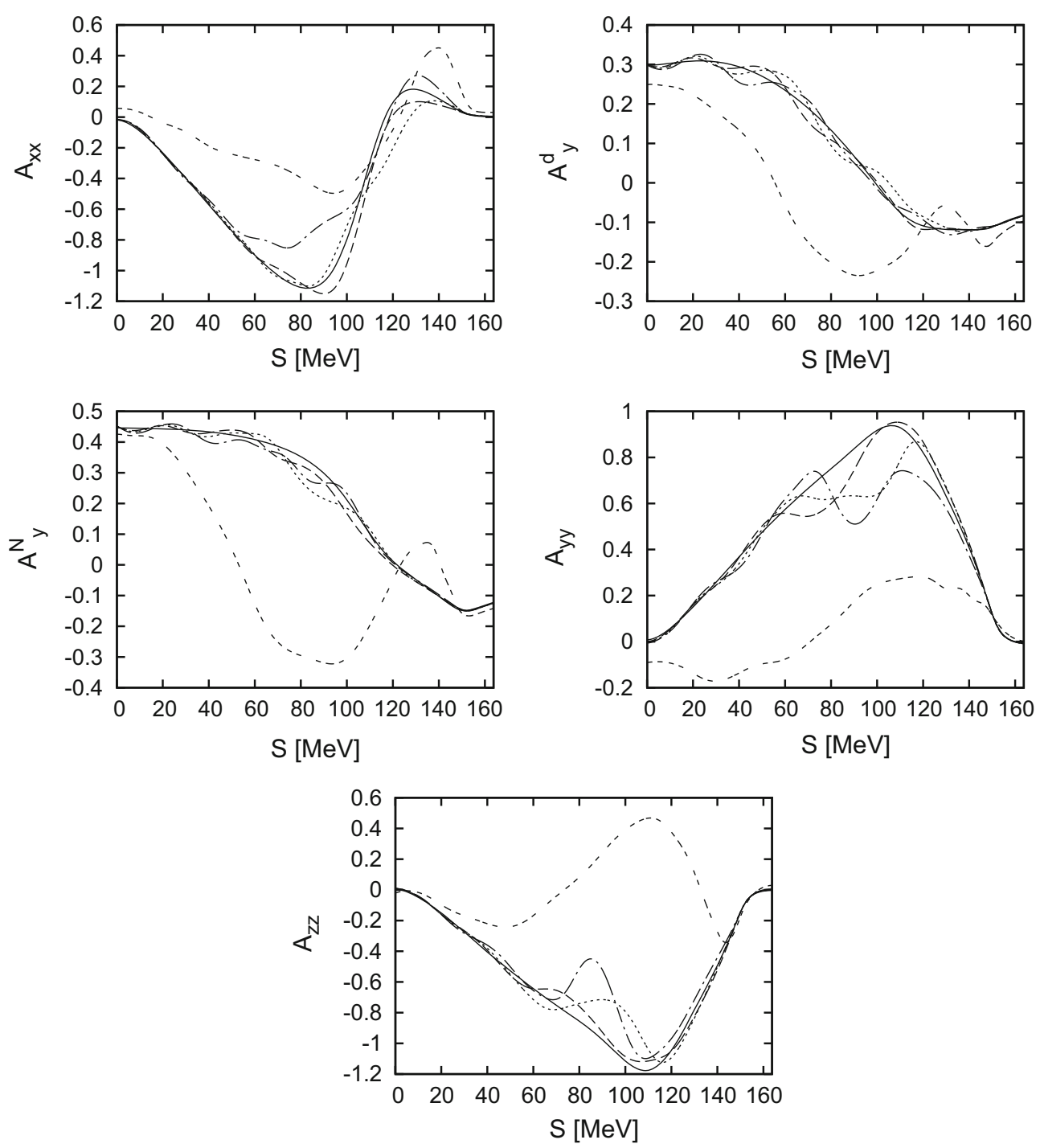

Fig. 1 Results from [3] for the deuteron and nucleon vector analyzing powers $A_{y}^{d}, A_{y}^{N}$ and the deuteron tensor analyzing powers $A_{x x}, A_{y y}, A_{z z}$ calculated for the $n d$ breakup reaction at laboratory kinetic energy $190 \mathrm{MeV}$. The $S$ on the vertical axes parametrize the kinematics of three free particles in the final state (details on the kinematics of the two neutrons and proton can be found in [3]). The dash-dotted, dotted and dashed lines are calculated using partial wave decomposition with maximum total angular momentum $21 / 2,23 / 2,25 / 2$, respectively and with maximum angular momentum of the 2-3 particle subsystem equal 8 . The solid lines are calculated using the 3D approach. The double dashed lines, for comparison, show partial wave results obtained using all order of the Faddeev equation (1). Convergence to the 3D results is observed in all plots, however in order to obtain full agreement more partial waves should be taken into account - this was unpractical with the computing resources used for the calculation

symmetries. Having the general operator form of $\check{T}|\phi\rangle$, the Faddeev equation can be transformed into a set of coupled linear equations for the scalar functions that define the scattering state. These equations could then be solved using methods familiar from $2 \mathrm{~N}$ transition operator calculations.

Acknowledgements The project was financed from the resources of the National Science Center (Poland) under Grants No. DEC-2013/11/N/ST2/03733 and DEC-2013/10/M/ST2/00420. Some of the numerical work was performed on the computers of JSC Jülich.

Open Access This article is distributed under the terms of the Creative Commons Attribution 4.0 International License (http:// creativecommons.org/licenses/by/4.0/), which permits unrestricted use, distribution, and reproduction in any medium, provided you give appropriate credit to the original author(s) and the source, provide a link to the Creative Commons license, and indicate if changes were made. 


\section{References}

1. W. Glöckle et al., The three-nucleon continuum: achievements, challenges and applications. Phys. Rep. 107, $274-285$ (1996)

2. W. Glöckle, The Quantum Mechanical Few-Body Problem (Springer, Berlin, Heidelberg, 1983)

3. K. Topolnicki et al., First-order neutron-deuteron scattering in a three-dimensional approach. Eur. Phys. J. A 51, 132 (2015)

4. J. Golak et al., Different methods for the two-nucleon T-matrix in the operator form. Few-Body Syst. 53, 237 (2012)

5. J. Golak et al., Two-nucleon systems in three dimensions. Phys. Rev. C 81, 034006 (2010)

6. L. Wolfenstein, Possible triple-scattering experiments. Phys. Rev. 96, 1654 (1954)

7. Y. Saad, Iterative Methods for Sparse Linear Systems (SIAM, Philadelphia, PA, 2003)

8. K. Topolnicki et al., The general operator form for the total-momentum-dependent nucleon-nucleon potential. Eur. Phys. J. A 52, $188(2016)$ 